\title{
TOXICIDAD DE PAULLINIACLAVIGERA SCHLTDL. (SAPINDACEAE) Y CHONDRODENDRON TOMENTOSUM RUIZ ET PAV. (MENISPERMACEAE) SOBRE EL PIOJO SALTADOR DEL CAMU CAMU TUTHILLIACOGNATA (HEMIPTERA: PSYLLIDAE)
}

\author{
TOXICITY OF PAULLINIA CLAVIGERA SCHLTDL. (SAPINDACEAE)AND \\ CHONDRODENDRON TOMENTOSUM RUIZ ET PAV. (MENISPERMACEAE) ON \\ JUMPING LICE OF CAMU CAMUTUTHILLIACOGNATA \\ (HEMIPTERA: PSYLLIDAE)
}

\author{
Diana Pérez ${ }^{1}$, José Iannacone ${ }^{2}$ \& Alfredo Tueros ${ }^{1}$ \\ ${ }^{1}$ Instituto de Investigaciones de la Amazonía Peruana (IIAP), Pucallpa, Ucayali, Perú; ${ }^{2}$ Laboratorio de Ecofisiología \\ Animal - Facultad de Ciencias Naturales y Matemáticas, Universidad Nacional Federico Villarreal, Perú. \\ joseiannacone@yahoo.es
}

\begin{abstract}
RESUMEN
Se determinó el efecto tóxico de dos extractos hidroalcohólicos procedentes de dos plantas amazónicas de importancia etnobotánica, Paullinia clavigera Schltdl. “sachayoco” (Sapindaceae) y Chondrodendron tomentosum Ruiz et Pav. "curare” (Menispermaceae), ambas en desarrollo vegetativo, sobre ninfas del III estadio de Tuthillia cognata Hodkinson, Brown \& Burckhardt, 1986 “piojo saltador del camu camu” (Hemiptera: Psyllidae). Las ninfas de T. cognata se colectaron en plantaciones del caserío San Juan en el distrito de Yarinacocha, Ucayali, Perú. Los bioensayos se realizaron empleando un Diseño de Bloque Completo al Azar (DBCA): 6 x 4. Los extractos se maceraron durante siete días a una proporción 1:10 (p/v) con posterior evaporación del solvente en rotavapor. El efecto tóxico se evaluó a 1, 4, 8, 12 y 24 h sobre $T$. cognata. En sachayoco se obtuvo una $\mathrm{CL}_{50-24 \mathrm{~h}}$ de $2.530 \mathrm{mg}$ de extracto $\mathrm{L}^{-1} \mathrm{y}$ en curare una $\mathrm{CL}_{50-24 \mathrm{~h}}$ de 4.090 mg de extracto $\mathrm{L}^{-1}$ sobre $T$. cognata. A una concentración de $10.000 \mathrm{mg}$ de extracto $\mathrm{L}^{-1}$, se determinó el $\mathrm{TL}_{50}$ del sachayoco y del curare a las 10,38 y 15,01 h, respectivamente. El análisis fitoquímico reveló saponinas, fenoles, flavonoides, quinonas y cumarinas en el extracto de sachayoco, así como alcaloides y saponinas en el extracto de curare. La toxicidad en términos de la $\mathrm{CL}_{50}$ dependió del tipo de extracto empleado.
\end{abstract}

Palabras clave: Bioensayo, fitoquímica, insecticida vegetal, Tuthillia cognata.

\begin{abstract}
Toxic effects of two hidro-alcoholical extracts belonging to two Amazonian plants of ethnobotanical importance, soapberry Paullinia clavigera Schltdl. (Sapindaceae) and curare Chondrodendron tomentosum Ruiz et Pav. (Menispermaceae), both in vegetative growing, on III nymph instars of jumping lice of camu camu Tuthillia cognata Hodkinson, Brown \& Burckhardt, 1986 (Hemiptera: Psyllidae) were determined. Collection of nymphs of T. cognata was performed on plantations of San Juan small town, Yarinacocha district, Ucayali, Peru. Bioassays were done employing a randomized completely block design (RCBD): 6 x 4. Maceration of extracts were performed during seven days at a proportion 1:10 $(\mathrm{w} / \mathrm{v})$ with a posterior solvent evaporation in a rotavapor. Toxic effect on $T$. cognata was evaluated at 1, 4, 8, 12 and 24 h. In soapberry $\mathrm{LC}_{50-24 \mathrm{~h}}$ was $2,530 \mathrm{mg}$ extract $\mathrm{L}^{-1}$ and in curare $\mathrm{LC}_{50-24 \mathrm{~h}}$ was $4,090 \mathrm{mg}$ extract $\mathrm{L}^{-1}$ on T. cognata. At a concentration of $10,000 \mathrm{mg}$ extract $\mathrm{L}^{-1}, \mathrm{LT}_{50}$ of soapberry and curare were 10.38 and $15.01 \mathrm{~h}$, respectively. Phytochemical analysis showed saponins, phenols, flavonoids, quinones and cumarins in soapberry extract, and alkaloids and saponins in curare extract. Toxicity in $\mathrm{LC}_{50}$ terms depended of the extract type employed.
\end{abstract}

KEYwords: Bioassays, phytochemical, insecticide plant, Tuthillia cognata. 


\section{INTRODUCCION}

El camu camu, Myrciaria dubia (Kunth) McVaugh, frutal nativo de la amazonia peruana, ha despertado gran interés en la agroindustria nacional e internacional debido a que sus frutos tienen alto contenido de acido ascórbico (2.000 a 3.000 mg·100 $\mathrm{g}^{-1}$ de pulpa fresca) (Penn 2006). La tendencia actual del camu camu es su producción orgánica, lo cual constituiría un producto ecológico de gran demanda dispuesto a satisfacer las exigencias mundiales del mercado agroindustrial. El manejo del cultivo radica en emplear técnicas de control integrado de plagas con la finalidad de erradicar insectos de importancia económica y evitar así mayores problemas fitosanitarios (Couturier et al. 1992, Iannacone et al. 2007b). Tuthillia cognata Hodkinson, Brown \& Burckhardt, 1986 (Hemiptera: Psyllidae) “piojo saltador del camu camu” en estado ninfal es una de las cuatro principales plagas del camu camu. Delgado \& Couturier (2004) y Penn (2006) mencionan a T. cognata en Iquitos, Jenaro Herrera, Requena, Mazan y Pucallpa, en Manaus en la amazonia brasileña, particularmente en ambientes de suelos inundables y no inundables donde se desarrolla el camu camu. Dicho insecto ocasiona daños en plantaciones jóvenes y en época de verano, a nivel de brotes foliares, disminuye y limita el desarrollo de las plantas y reduce la capacidad de fotosíntesis de aquellas en producción (> 3 años) (Burckhardt \& Couturier 1988, Delgado \& Couturier 2004). La plaga produce infestaciones entre 12 y $94 \%$ en plantas en desarrollo, siendo la parte superior la preferida por el insecto (68\%) (Delgado \& Couturier 2004). Además provoca deformaciones en hojas jóvenes, impidiendo el crecimiento de brotes. Al comienzo del ataque las hojas se ensanchan, a nivel de la nervadura principal; luego el brote se torna amarillo y se necrosa para posteriormente secarse (Burckhardt \& Couturier 1988, Delgado \& Couturier 2004). Las ninfas viven en el interior de la zona afectada donde forman colonias y succionan la savia de la hoja (Burckhardt \& Couturier 1988).

Con relación a sus enemigos naturales, existen dos depredadores de $T$. cognata, Ocyptamus persimilis Curran, 1930 (Diptera. Syrphidae) y una hormiga, Camponotus rufipes (Fabricius, 1775) (Hymenoptera: Formicidae) que se alimentan de las ninfas de esta plaga (Couturier et al. 1992). Otras medidas de control recomiendan recoger con mucho cuidado los brotes infestados y posteriormente quemarlos. La recolección de brotes tiene que realizarse en baldes o bolsas plásticas, para evitar que las ninfas y adultos se escapen, o los huevos sean transportados por el viento a otras plantas o parcelas (Delgado \& Couturier 2004).

Por otra parte, las poblaciones naturales de Chondrodendron tomentosum Ruiz et Pav. "curare", se ubican en el Perú en ecosistemas de altura formando bosques primarios, donde requieren de poca luminosidad, altitudes de 150 a 2000 m s.n.m., suelos arcillosos, y tolerables a suelos con baja fertilidad (Gupta 1995). Las comunidades nativas Ashaninkas, emplean las raíces y tallos como tóxico en la punta de sus flechas para cazar animales (Duke \& Vásquez 1994). Del mismo modo, las poblaciones naturales de Paullinia clavigera Simpson "sacha yoco" se localizan en ecosistemas de altura formando parte del bosque primario, pero en planicies anegadizas requieren de poca luminosidad, de moderada a alta humedad relativa, altitudes de 150 a $2.000 \mathrm{~m}$ s.n.m., suelos arcillosos y tolerables a suelos con baja fertilidad (Gupta 1995). La raíz se utiliza como veneno para la pesca (ictiotóxico), y presenta actividad antifúngica y molusquicida (Pérez \& Iannacone 2006).

Ch. tomentosum y P. clavigera son dos especies vegetales promisorias de la amazonia que se vienen estudiando para el control de las principales plagas de los cultivos amazónicos, con efecto tóxico, biocida y fagodisuasivo. Por lo tanto, el uso de plantas biocidas constituye una de las alternativas más alentadoras para el control de plagas, por su bajo costo, fácil disponibilidad y bajo impacto al medio ambiente en condiciones de aplicación controlada (Bobadilla et al. 2002, Iannacone et al. 2002, 2007a, Xiaorong \& Taiping 2008).

Sin embargo, en la amazonia peruana pese a su gran biodiversidad y gran potencial de plantas biocidas, se ha efectuado pocas investigaciones al respecto. Bajo las consideraciones anteriores, es necesario incrementar una base de datos respecto a la identificación de plantas biocidas provenientes de la amazonia peruana, con énfasis en el control de insectos plagas de cultivos de importancia agroindustrial, razones fundamentales para determinar el efecto tóxico insecticida de dos extractos de las plantas Ch. tomentosum y P. clavigera sobre el tercer estadio ninfal de T. cognata (Hemiptera: Psyllidae) plaga principal del cultivo de camu camu. 
Toxicidad de Paullinia y Chondrodendron en Tuthillia: PéreZ ET AL.

\section{MATERIALES Y METODOS}

LUGAR DE EJECUCIÓN Y FACTORES AMBIENTALES

El presente trabajo de investigación se desarrolló desde agosto a diciembre del 2006 en el Laboratorio de Entomología del Instituto de Investigaciones de la Amazonia Peruana (IIAP), localizado en el km 12.400 en el margen derecho de la Carretera Federico Basadre (CFB), en el Distrito de Callería, Provincia de Coronel Portillo, Región Ucayali, Perú (8²2’31’S, 74 34’23” W) a una altitud de 154 m s.n.m. La zona pertenece al ecosistema mayor de Bosque Tropical Semi Siempre Verde Estacional. Según la estación meteorológica de CORPAC-Ucayali, Perú, en los últimos diez años (1993-2002) se registró los siguientes datos: temperatura media anual de 25,5 ${ }^{\circ} \mathrm{C}$, humedad relativa de $83 \%$ y precipitación anual de $1.452 \mathrm{~mm}$.

TUTHILLIA COGNATA

La recolección de ninfas de $T$. cognata se realizó en plantaciones de camu camu del Caserío San Juan en el distrito de Yarinacocha, en las parcelas de la empresa Agrícola San Juan ubicada en el km 13 de la CFB, y en la estación del IIAP. Se consideraron estos dos lugares representativos para la recolección debido a la elevada densidad del insecto plaga en dichas plantaciones. La captura se realizó a partir de las 5:30 AM, en forma manual, extrayendo las hojas con síntomas y presencia de ninfas de $T$. cognata del II estadio a punto de mudar a ninfas del III estadio. Luego se procedió a depositarlas en envases de plástico de 15 x $20 \mathrm{~cm}$ acondicionados con un forro interior de papel toalla y una abertura en la cubierta forrada con tela de organza ${ }^{\circledR}$ que permitió el ingreso de aire. Posteriormente los envases fueron llevados al laboratorio, en donde se seleccionaron las ninfas para los bioensayos toxicológicos (Pérez \& Iannacone 2006).

\section{EXTRACTOS}

Se maceraron durante 7 días en un depósito de vidrio ámbar y en ambiente oscuro, 3L de etanol al 96\% con 300 g de hojas de Ch. tomentosum y $300 \mathrm{~g}$ del tallo de $P$. clavigera, ambos en desarrollo vegetativo manteniendo una relación cuantitativa de 1:10 (100 g de biomasa seca de cada planta por litro de solvente). En este periodo de tiempo, el aumento de la capacidad de maceración y homogeneidad de la muestra con el solvente, se realizó con agitación diaria del frasco por 15 segundos. Luego se procedió a la filtración con tela de organza, obteniéndose 2.160 $\mathrm{ml}$ de extracto de Ch. tomentosum de color verde cristalino y $2.250 \mathrm{ml}$ de extracto de $P$. clavigera de color rojo tinto. La concentración de cada extracto se realizó empleando un equipo rotavapor (Laborota 4000- Efficient, marca Heldolph Instrument ${ }^{\circledR}$, Alemania) con rotación permanente de 150 rpm para Ch. tomentosum y $180 \mathrm{rpm}$ para $P$. clavigera, el condensador o refrigerante a $10^{\circ} \mathrm{C}$, el vacío aplicado fue de $600 \mathrm{mmHg}$ de presión y la temperatura de 50 ${ }^{\circ} \mathrm{C}$. Este proceso duró $2 \mathrm{~h} 20 \mathrm{~min}$ para Ch. tomentosum y 2 h 55 min para $P$. clavigera. Posteriormente, los extractos secos se redisolvieron en agua destilada con lo que se obtiene el extracto madre.

\section{Metabolitos SECUndarios}

Procedimientos estándares para la detección fitoquímica semicualitativa de nueve compuestos (Lock 1994) se emplearon para alcaloides (Mayer, Dragendorff y Wagner), saponinas (producción de espuma), esteroides (Liebermann Burchard), triterpenos (Liebermann-Burchard/Noller), taninos (gelatina-sal $/ \mathrm{FeCl}_{3}$ ), fenoles $\left(\mathrm{FeCl}_{3}\right)$, flavonoides (Shinoda), cumarinas (revelado con vainilla y ácido ortofosfórico) y quinonas (Bornträger). Se usó el índice de Sörensen para determinar el grado de similaridad entre los principales grupos funcionales fitoquímicos de ambos extractos etanólicos (Moreno 2001).

Preparación de Las SOluciones CONCENTRAdas y DILUCIONES (Iannacone et al. 2007a)

Para obtener la solución concentrada de $C h$. tomentosum y de P. clavigera se pesó 2 g del extracto madre y se diluyó en 200 ml de agua destilada, a partir de esta solución se realizaron diluciones para los tratamientos, a partir de 62,5 mg; 125; 250; 500 y 1.000 g, diluidos en $100 \mathrm{ml}$ de agua destilada, respectivamente. Se distribuyó en aspersores manuales de $150 \mathrm{ml}$ de capacidad para su posterior aplicación.

\section{Bioensayos}

La captura de ninfas de T. cognata, tuvo por finalidad la obtención de especímenes para la realización de los bioensayos, la cual se realizó momentos antes de iniciar las pruebas. Las ninfas del III estadio se transfirieron a envases de plástico descartables de 6 cm de altura por 11,5 cm de diámetro en número de uno por envase, sobre una hoja tierna de camu camu. Las condiciones y criterios de aceptabilidad de la prueba de toxicidad aguda para $T$. cognata (Iannacone \& Lamas 2003) fueron: 
Gayana Bot. 65(2), 2008

-Tipo de bioensayo: estático.

- Tiempo de exposición: 1, 4, 8, 12 y 24 h.

- Temperatura: $30 \pm 3{ }^{\circ} \mathrm{C}$.

- Humedad relativa: 60 -85\%.

-pH de solución: 7.

-Fotoperiodo: 12:12.

-Volumen de aspersión manual: 0,23 ml por individuo.

-Edad de organismos: III estadio ninfal.

-Número de tratamientos: 6.

-Número de réplicas por tratamiento: 4.

-Número de ninfas por repetición: 10.

-Número de ninfas por tratamiento: 40.

-Número total de ninfas por ensayo: 240.

-Número de organismos por envase: 1.

-Régimen de alimentación: brotes de camu camu.

-Agua control y de dilución: destilada.

-Tiempo de observación: 10 seg de observación directa.

-Respuesta letal: mortalidad (cuando no reaccionan al ser tocadas con un pincel por espacio de $10 \mathrm{seg}$ ).

-Criterio de aceptabilidad sugerida sobre $80 \%$ de sobrevivencia en los controles.

Las pruebas de toxicidad aguda de los dos extractos sobre $T$. cognata se evaluaron en cinco concentraciones más un control, con cuatro repeticiones, en un Diseño de Bloque Completo al Azar (DBCA) de 6 x 4 (Iannacone \& Lamas 2003).
ANÁLISIS ESTADÍsTICO

La eficacia de los tratamientos y las repeticiones se evaluó a través de un análisis de varianza (ANDEVA) de dos vías, previa transformación de los datos a $\sqrt{\mathrm{x}}+0,5$. En el caso de existir diferencias significativas entre los tratamientos y las repeticiones se realizó la prueba de Tukey. Los cálculos de la mortalidad corregida se realizaron mediante la fórmula de Abbott en caso de muerte natural en el grupo testigo cuando éste era menor al 20\% (Calderón \& Carvalho 2001). Los datos obtenidos se analizaron mediante el paquete SPSS versión 12 (SPSS 2004). Las concentraciones letales medias $\left(\mathrm{CL}_{50}\right)$ y los tiempos letales medias $\left(\mathrm{TL}_{50}\right)$ se determinaron utilizando el EPA ProbitAnalysis Program (USEPA 1994).

\section{RESULTADOS}

No se observaron diferencias significativas en los porcentajes de mortalidad de $T$. cognata por acción de los extractos de Ch. tomentosum y P. clavigera, en ninguna de las cinco concentraciones (Tablas I y II). A 4 y 8 h de exposición en Ch. tomentosum se observa mortalidad diferente al control a $10.000 \mathrm{mg}$ de extracto $\mathrm{L}^{-1}$ (Tabla I). De igual forma para $C h$. tomentosum a 12 y 24 h de exposición, se observa

TABLA I. Efecto tóxico de Chondrodendron tomentosum en la mortalidad de ninfas de Tuthillia cognata en bioensayos de laboratorio a diferentes periodos de exposición.

TABLE I. Toxicological effect of Chondrodendron tomentosum on mortality of Tuthillia cognata nymphs in laboratory bioassays to different periods of exposure.

\begin{tabular}{|c|c|c|c|c|c|c|c|c|c|c|c|}
\hline \multirow{2}{*}{$\begin{array}{l}\text { Concentración } \\
\mathrm{mg} \cdot \mathrm{L}^{-1}\end{array}$} & \multicolumn{2}{|c|}{$1 \mathrm{~h}$} & \multicolumn{2}{|c|}{$4 \mathrm{~h}$} & \multicolumn{2}{|c|}{$8 \mathrm{~h}$} & \multicolumn{2}{|c|}{$12 \mathrm{~h}$} & \multicolumn{2}{|c|}{$24 \mathrm{~h}$} & \multirow[b]{2}{*}{$\mathrm{TL}_{50}$ (h). } \\
\hline & Mort. & Sig. & Mort. & Sig. & Mort. & Sig. & Mort. & Sig. & Mort. & Sig. & \\
\hline Agua destilada & 0 & $\mathrm{a}$ & 0 & b & 0 & $\mathrm{~b}$ & 2,5 & c & 5 & b & ND \\
\hline 625 & 0 & $\mathrm{a}$ & 0 & b & 0 & $\mathrm{~b}$ & 2,5 & bc & 12,5 & b & 53,59 \\
\hline 1250 & 0 & $\mathrm{a}$ & 0 & b & 0 & $\mathrm{~b}$ & 5 & $\mathrm{abc}$ & 17,5 & $\mathrm{~b}$ & 46,53 \\
\hline 2500 & 0 & $\mathrm{a}$ & 0 & b & 2,5 & $\mathrm{~b}$ & 10 & $a b c$ & 42,5 & $\mathrm{a}$ & 26,83 \\
\hline 5000 & 0 & a & 2,5 & $a b$ & 5 & $\mathrm{~b}$ & 17,7 & $\mathrm{ab}$ & 60 & a & 21,27 \\
\hline 10000 & 0 & a & 7,5 & a & 20 & $\mathrm{a}$ & 35 & $\mathrm{a}$ & 75 & a & 15,01 \\
\hline $\mathrm{F}$ & ND & & 4,71 & & 6,78 & & 4,84 & & 21,22 & & \\
\hline Sig. & ND & & 0,008 & & 0.001 & & 0,007 & & 0,0001 & & \\
\hline $\mathrm{CL}_{50}\left(\mathrm{mg} \cdot \mathrm{L}^{-1}\right)$ & ND & & ND & & ND & & 18334 & & 4090 & & \\
\hline
\end{tabular}

Promedios en una misma línea vertical seguidos por la misma letra minúscula no difieren significativamente a $\mathrm{P}=0,05$.

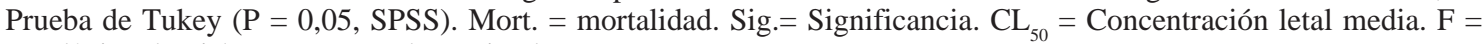
estadístico de Fisher. ND = No determinado.

Average in the same vertical line followed by the same lower case letter not indicated significant differences at $\mathrm{P}=0.05$. Tukey's test $\left(\mathrm{P}=0.05\right.$, SPSS). Mort. = mortality. Sig. = significance; $\mathrm{LC}_{50}=$ median lethal concentration. $\mathrm{F}=$ Fisher's statistical. ND = Not determined. 
Toxicidad de Paullinia y Chondrodendron en Tuthillia: PéREZ eT AL.

TABla II. Efecto toxicológico de Paullinia clavigera en la mortalidad de ninfas de Tuthillia cognata en bioensayos de laboratorio a diferentes periodos de exposición.

TABLE II. Toxicological effect of Paullinia clavigera on mortality of Tuthillia cognata nymphs in laboratory bioassays to different periods of exposure.

\begin{tabular}{|c|c|c|c|c|c|c|c|c|c|c|c|}
\hline \multirow{2}{*}{$\begin{array}{l}\text { Concentración } \\
\mathrm{mg} \cdot \mathrm{L}^{-1}\end{array}$} & \multicolumn{2}{|c|}{$1 \mathrm{~h}$} & \multicolumn{2}{|c|}{$4 \mathrm{~h}$} & \multicolumn{2}{|c|}{$8 \mathrm{~h}$} & \multicolumn{2}{|c|}{$12 \mathrm{~h}$} & \multicolumn{2}{|c|}{$24 \mathrm{~h}$} & \multirow[b]{2}{*}{$\mathrm{TL}_{50}(\mathrm{~h})$} \\
\hline & Mort. & Sig. & Mort. & Sig. & Mort. & Sig. & Mort. & Sig. & Mort. & Sig. & \\
\hline Agua destilada & 0 & $\mathrm{a}$ & 0 & $\mathrm{~b}$ & 0 & c & 0 & b & 5 & $\mathrm{~b}$ & ND \\
\hline 625 & 0 & a & 0 & b & 0 & c & 22,5 & $a b$ & 25 & b & 38,36 \\
\hline 1250 & 0 & a & 0 & b & 2,5 & bc & 27,5 & $a b$ & 30 & $\mathrm{~b}$ & 33,07 \\
\hline 2500 & 0 & $\mathrm{a}$ & 0 & $\mathrm{~b}$ & 20 & $a b$ & 42,5 & $\mathrm{ab}$ & 55 & $\mathrm{ab}$ & 18,07 \\
\hline 5000 & 0 & a & 2,5 & $\mathrm{~b}$ & 22,5 & $\mathrm{abc}$ & 50 & $\mathrm{a}$ & 62,5 & $\mathrm{ab}$ & 15,34 \\
\hline 10000 & 0 & a & 5 & $\mathrm{~b}$ & 30 & a & 65 & $\mathrm{a}$ & 87,5 & a & 10,62 \\
\hline $\mathrm{F}$ & ND & & 0,79 & & 7,12 & & 4,05 & & 5,77 & & \\
\hline Sig. & ND & & 0,57 & & 0,001 & & 0,015 & & 0,003 & & \\
\hline $\mathrm{CL}_{50}\left(\mathrm{mg} \cdot \mathrm{L}^{-1}\right)$ & ND & & ND & & ND & & 4366 & & 2530 & & \\
\hline
\end{tabular}

Promedios en una misma línea vertical seguidos por la misma letra minúscula no difieren significativamente a $\mathrm{P}=0,05$.

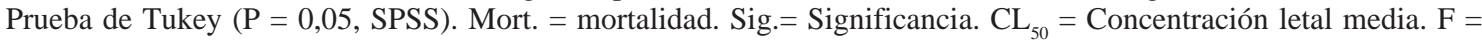
estadístico de Fisher. ND = No determinado.

Average in the same vertical line followed by the same lower case letter not indicated significant differences at $\mathrm{P}=0.05$. Tukey's test $(\mathrm{P}=0.05$, SPSS $)$. Mort. = mortality. Sig. = significance; $\mathrm{LC}_{50}=$ median lethal concentration. $\mathrm{F}=$ Fisher's statistical. ND = Not determined.

mortalidad significativa de $T$. cognata desde 5.000 y $2.500 \mathrm{mg}$ de extracto $\mathrm{L}^{-1}$, respectivamente (Tabla I). $\mathrm{La} \mathrm{CL}_{50}$ de Ch. tomentosum tiende a incrementar su toxicidad de 12 a 24 h; así mismo, el $\mathrm{TL}_{50}$ disminuye numéricamente de 625 a $10.000 \mathrm{mg}$ de extracto $\mathrm{L}^{-1}$ (Tabla I).

A 4 h de exposición en $P$. clavigera no se observa mortalidad diferente al control a ninguna de las concentraciones de este extracto (Tabla II). De igual forma para P. clavigera a 8,12 y 24 h de exposición, se observa mortalidad significativa de T. cognata entre 2.500 y 10.000 mg de extracto $\mathrm{L}^{-1}$ (Tabla II). La $\mathrm{CL}_{50}$ de $P$. clavigera tiende a incrementar su toxicidad de 12 a 24 h; así mismo, el $\mathrm{TL}_{50}$ disminuye numéricamente de 625 a $10.000 \mathrm{mg}$ de extracto $\mathrm{L}^{-1}$ (Tabla II).

La $\mathrm{CL}_{50}$ de $P$. clavigera fue 4,2 y 1,6 veces mas sensible que la $\mathrm{CL}_{50}$ de $C h$. tomentosum para $T$. cognata a 12 y 24 h de exposición, respectivamente (Tablas I y II). De igual manera, el $\mathrm{TL}_{50}$ de $P$. clavigera fue 1,3 y 1,4 veces más sensible que el $\mathrm{TL}_{50}$ de $C h$. tomentosum para T. cognata a 5.000 y $10.000 \mathrm{mg}$ de extracto $\mathrm{L}^{-1}$, respectivamente (Tablas I y II).

Con relación a las principales características fitoquímicas de los extractos etanólicos de $C h$. tomentosum y P. clavigera, se encontró en el primero alcaloides, saponinas, cumarinas y quinonas; y en el segundo extracto se observó saponinas, fenoles, flavonoides, cumarinas y quinonas (Tabla III). Se encontró una similaridad de 66,66\% entre ambos extractos con relación a sus principales grupos funcionales fitoquímicos, según el índice de Sörensen.

\section{DISCUSION}

Pérez \& Iannacone (2006, 2008) al evaluar el efecto integral de diez extractos acuosos de plantas, encontraron para la mortalidad en general, un aumento en el tiempo de exposición. Este patrón también fue observado en el presente estudio.

Calvo (2006) menciona que las saponinas, desde el punto de vista biológico, pueden tener efectos perjudiciales, actuando como antinutrientes, o tóxicos; su alta capacidad tensoactiva altera las membranas celulares, y en el tubo digestivo no se absorben, por lo que su efecto produciría alteraciones de membranas, y posiblemente aumentaría su permeabilidad. Philogene (2004) indica que cualquier sustancia cuya acción facilite o estimule la penetración, el transporte o la accesibilidad de un compuesto tóxico, debe 
Gayana Bot. 65(2), 2008

TABLA III. Análisis fitoquímicos de los extractos etanólicos de Chondrodendron tomentosum y Paullinia clavigera.

TABLE III. Phytochemical analysis of ethanolic extracts of Chondrodendron tomentosum and Paullinia clavigera.

\begin{tabular}{lcc}
\hline Pruebas fitoquímicas & $\begin{array}{c}\text { Chondrodendron } \\
\text { tomentosum }\end{array}$ & $\begin{array}{c}\text { Paullinia } \\
\text { clavigera }\end{array}$ \\
\hline Masa a partir 100 g (en g) & 13,07 & 8,75 \\
Alcaloides & +++ & - \\
Saponinas & ++ & +++ \\
Esteroides & - & - \\
Triterpenos & - & - \\
Taninos & - & - \\
Fenoles & - & ++ \\
Flavonoides & - & ++ \\
Cumarinas & + & ++ \\
Quinonas & \pm & ++ \\
\hline
\end{tabular}

Leyenda: - : reacción negativa. \pm : reacción muy poco positiva. + : reacción poco positiva. ++ : reacción positiva. +++ : reacción muy positiva.

Legend: - : negative reaction. \pm : very low positive reaction. + : low positive reaction. ++ : positive reaction. +++ : very positive reaction.

considerarse como un “casi sinergista”, éste es el caso de las saponinas que favorecen la penetración de otros productos tóxicos en las células; siendo probable el ingreso de los alcaloides de $C h$. tomentosum a T. cognata mediante esta acción. Los alcaloides presentes en el extracto de $C h$. tomentosum probablemente produzcan parálisis en T. cognata, al actuar sobre el sistema nervioso central. Schultes \& Raffauf (1990) y Desmarchelier \& Witting (2000) mencionan que la actividad insecticida del curare se debe a los alcaloides isoboldina y Dtubocurarina.

La alta mortalidad observada por P. clavigera sobre T. cognata probablemente está influenciada por sus principios activos. Gupta (1995) menciona que la toxicidad de esta especie es producida por triterpenos y saponinas. Asimismo se menciona la presencia de triterpenos, B-sitosterol y de aceites etéreos, atribuyéndoles a los primeros la actividad ictiotóxica (Gupta 1995, Iannacone et al. 2007a). Ekabo et al. (1996) señalan que las propiedades antifúngicas y molusquicidas de $P$. clavigera son debidas a las saponinas, lo cual concuerda con las altas concentraciones de este grupo funcional fitoquímico encontrado en el extracto etanólico (Tabla III). Asimismo, Pérez \& Iannacone (2004) señalan propiedades insecticidas del extracto acuoso de $P$. clavigera sobre larvas del tercer estadio de Anopheles benarrochi Gabaldon, Cova García y López, 1941 (Diptera: Culicidae).
P. clavigera es mas tóxica que Ch. tomentosum sobre $T$. cognata, tanto en términos de $\mathrm{CL}_{50} \mathrm{y} \mathrm{TL}_{50}$. Pérez \& Iannacone (2006, 2008), utilizando diez extractos acuosos sobre Rhynchophorus palmarum (Linne, 1764) (Coleoptera: Curculionidae) y Eupalamides cyparissias (Fabricius, 1777) (Lepidoptera: Castniidae) plagas del pijuayo para palmito (Bactris gassypaes Kunth) y la palma aceitera (Elaeis guineensis Jacq.) respectivamente, obtuvieron los mayores resultados con el extracto de $P$. clavigera con $63,3 \%$ de mortalidad en comparación con Ch. tomentosum, en el que se observó 8,3\% de mortalidad en larvas de $E$. cyparissias. Contrariamente, en el caso de $R$. palmarum el extracto de Ch tomentosum obtuvo un $60 \%$ y el de $P$. clavigera un $31,7 \%$ de mortalidad.

La actividad tóxica de los principios activos de las dos plantas biocidas empleadas sería principalmente de contacto, mediante tres mecanismos interdependientes: transporte desde la cutícula al sitio de acción, inhibición enzimática y efecto sobre el sistema nervioso central, respiratorio u otro sistema involucrado como una consecuencia bioquímica del primer mecanismo (Isman 2006). Entre los factores inherentes al organismo de prueba, es de destacar la variación de la susceptibilidad de acuerdo a la edad, estado de desarrollo, reorganización anatómica y a las variaciones propias de la muda (Silva \& Casals 1994). De esta forma se prefirió trabajar con el III estadio ninfal de T. cognata. 
Rodríguez (2000) sostiene que las sustancias naturales de las plantas no son tan agresivas ni fulminantes como los insecticidas convencionales, de aquí que este autor no los considere insecticidas sino insectistáticos.

La evaluación de la biodiversidad de los bosques tropicales amazónicos revela nuevos principios biocidas, pero estos estudios aún están en su inicio. La biodiversidad existente en la flora peruana es una fuente potencial de moléculas nuevas bioactivas. Los extractos de plantas más eficientes deben ser priorizados para el fraccionamiento e identificación de sus principales componentes activos (Kumar et al. 2007, Moreira et al. 2007).

\section{BIBLIOGRAFIA}

Bobadilla, M., G. Zavaleta, G. Franco \& L. Pollack. 2002. Efecto bioinsecticida del extracto etanólico de las semillas de Annona cherimolia Miller “chirimoya” y A. muricata Linneaeus "guanábana” sobre larvas del IV estadio de Anopheles sp. Revista Peruana de Biología 9: 64-73.

Burckhardt, D. \& G. Couturier. 1988. Biology and taxonomy of Tuthillia cognata (Homoptera: Psyllidae), a pest on Myrciaria dubia. Annals de la Societé Entomologica Francaise 24: 257- 261.

Calderón, G. \& M. Carvalho. 2001. Pruebas de susceptibilidad de mosquitos adultos y larvas a los insecticidas y bioensayos de las aplicaciones residuales y espaciales usadas en el control de mosquitos vectores de malaria y dengue. STC, OPS/OMS. Paraiba, Brasil. 55 pp.

Calvo, M. 2006. Bioquímica de los alimentos. Universidad de Zaragoza. España. (Accesado: Enero, 6, 2006). http://milksci.unizar.es/bioquimica/temas/toxico/ otrassubstancias.html

Couturier, G., H. Inga \& E. Tanchiva. 1992. Insectos fitófagos que viven en Myrciaria dubia (Myrtaceae) frutal amazónico de la región Amazónica. Folia Amazónica 4: 19-35.

Delgado, C. \& G. Couturier. 2004. Manejo de insectos plagas en la Amazonía: su aplicación en camu camu. IIAP- Iquitos. IRD- Francia. Lima, Perú. 147 pp.

Desmarchelier, C. \& F. Witting. 2000. Sesenta plantas medicinales de la amazonia peruana, ecología, etnomedicina y bioactividad. Turbera Inc. S.A., Lima, Perú. 270 pp.

Duke, J. A. \& R. VÁsquez. 1994. Amazonian Ethnobotanical Dictionary. CRC Press. USA. Inc. Boca Raton, Florida, USA. 215 pp.

Ekabo, O., N. Farnswoth, T. Henderson, G. Mao \& R. MukHerJeE. 1996. Antifungal and molluscicidal saponins from Serjania salzmanniana. Journal of Natural Products 59: 431-435.

Gupta, M.P. 1995. 270 Plantas Medicinales Iberoamericanas. Programa Iberoamericano de
Ciencia y Tecnología para el Desarrollo (CYTED). Santa Fé de Bogotá. Colombia. 441 pp.

IANNACONE, J. \& G. LAMAS. 2003. Efectos toxicológicos del nim, rotenona y cartap sobre tres microavispas parasitoides de plagas agrícolas en el Perú. Boletín de Sanidad Vegetal Plagas 29: 123-142.

Iannacone, J., L. Alvariño \& J. Mansilla. 2002. Actividad insecticida de cuatro extractos botánicos sobre larvas de los mosquitos Culex quinquefasciatus (Díptera: Culicidae) y Chironomus calligraphus (Díptera: Chironomidae). Wiñay Yachay 7: 56-71.

Iannacone, J., L. Alvariño, J.C. Soto \& C. SAlCEdo. 2007 a. Efecto toxicológico del "Sachayoco", Paullinia clavigera (Sapindaceae) sobre Daphnia magna y sobre dos controladores biológicos de plagas agrícolas. Journal of the Brazilian Society of Ecotoxicology 2: 15-25.

IAnnacone, J., D. Pérez \& A. Tueros. 2007b. Ciclo de vida y aspectos poblacionales de Edessa aff. aulacosterna Stal, 1872 (Heteroptera: Pentatomidae) chinche del fruto del camu camu (Myrtaceae) en zona de restinga, Ucayali, Perú. Acta Amazónica 37: 637-644.

IsmAN, M. I. 2006. Botanical insecticides, deterrents, and repellents in modern agriculture and an increasingly regulated world. Annual Review of Entomology 51: 45-66.

Kumar, R., M. Srivastava \& N.K. Dubey. 2007. Evaluation of Cymbopogon martinii oil extract for control of postharvest insect deterioration in cereals and legumes. Journal of Food Protection 70: 172-178.

Lock, O. 1994. Investigación Fitoquímica: métodos de estudio de productos naturales. Pontificia Universidad Católica del Perú. Fondo Editorial. Lima, Perú. 300 pp.

Moreira, M. D., M.C. Picanco, L.C.A. Barbosa, R. N. C. Guedes, M. Ribeiro, G. A. Silva \& J. C. Martins. 2007. Plant compounds insecticide activity against Coleoptera pests of stored products. Pesquisas agropecuarias brasileiras 42: 909-915.

Moreno, C. 2001. Métodos para medir la biodiversidad. M\&T-Manuales y Tesis SEA, vol. 1. Zaragoza, España. 84 pp.

Penn, J.W. JR. 2006. The cultivation of camu camu (Myrciaria dubia): a tree planting programme in the peruvian amazon. Forests, Trees and Livelihoods 16: 85-101.

Pérez, D. \& J. Iannacone. 2004. Efecto insecticida del Sacha yoco (Paullinia clavigera var. bullata Simpson) (Sapindaceae) y oreja de tigre (Tradescantia zebrina Hort ex Bossé) (Commelinaceae) en el control de Anopheles benarrochi Gabaldon, Cova García y López, 1941, principal vector de malaria en Ucayali, Perú. Ecología Aplicada 3: 64-72.

Pérez, D. \& J. IAnNacone. 2006. Efectividad de extractos botánicos de diez plantas sobre la mortalidad y repelencia de larvas de Rhynchophorus palmarum L., insecto plaga del pijuayo Bactris gasipaes Kunth en la Amazonía del Perú. Agricultura Técnica (Chile) 66: 21-30. 
Gayana Bot. 65(2), 2008

Pérez, D. \& J. IAnnacone. 2008. Mortalidad y repelencia en Eupalamides cyparissias (Lepidoptera: Castniidae), plaga de la palma aceitera Elaeis guineensis, por efecto de diez extractos botánicos. Revista de la Sociedad Entomológica Argentina 67: 41-48.

Philogene, B. J. R. 2004. Biopesticidas de origen vegetal. En: C. Regnault-Roger, B.J.R. Philogene \& C. Vincent (eds.), Acción sinérgica de los compuestos de origen vegetal. Mundi-Prensa. Madrid, España. 67-76.

Rodríguez, C. 2000. Plantas Contra Plagas: Potencial práctico de ajo, anona, nim, chile y tabaco. Ed. RAPAM. México. D.F. 133 pp.

Schultes, R.E. \& R.F. Raffaut. 1990. The Healing Forest. Medicinal and Toxic Plants of the Northwest Amazonia. Dioscorides Press. Portland, Oregon, USA. 484 pp.
Silva, G. \& P. Casals. 1994. Bioensayo. (Accesado: Marzo 14, 2002). http://www.multired.com./ciencia/ gosilagu/.

SPSS. 2004. Statistical Package for the Social Sciences (SPSS). SPSS Base 12.0 User's Guide for Windows. SPSS Inc., Chicago, Illinois, USA. Available at (Accesado: Diciembre 8, 2007). http:// www.spss.com//spss.

USEPA. 1994. User's guide: Probit Program version 1,5. U.S. Environmental Protection Agency (USEPA). Ecological Monitoring Research Division, Cincinatti, Ohio, USA.

Xiaorong, T. \& H. TAiping. 2008. Separation and identification of botanical insecticide 7 hydroxycoumarin and its biological activity against Aphis craccivora and Culex pipiens pallens. Natural Product Research 22: 365-370.

Recibo: 01.04 .08

Aceptado: 14.05 .08 